\title{
Capital social y participación en los programas sociales*
}

Arq. Aldo de la Puente

Docente investigador FADU/UNL

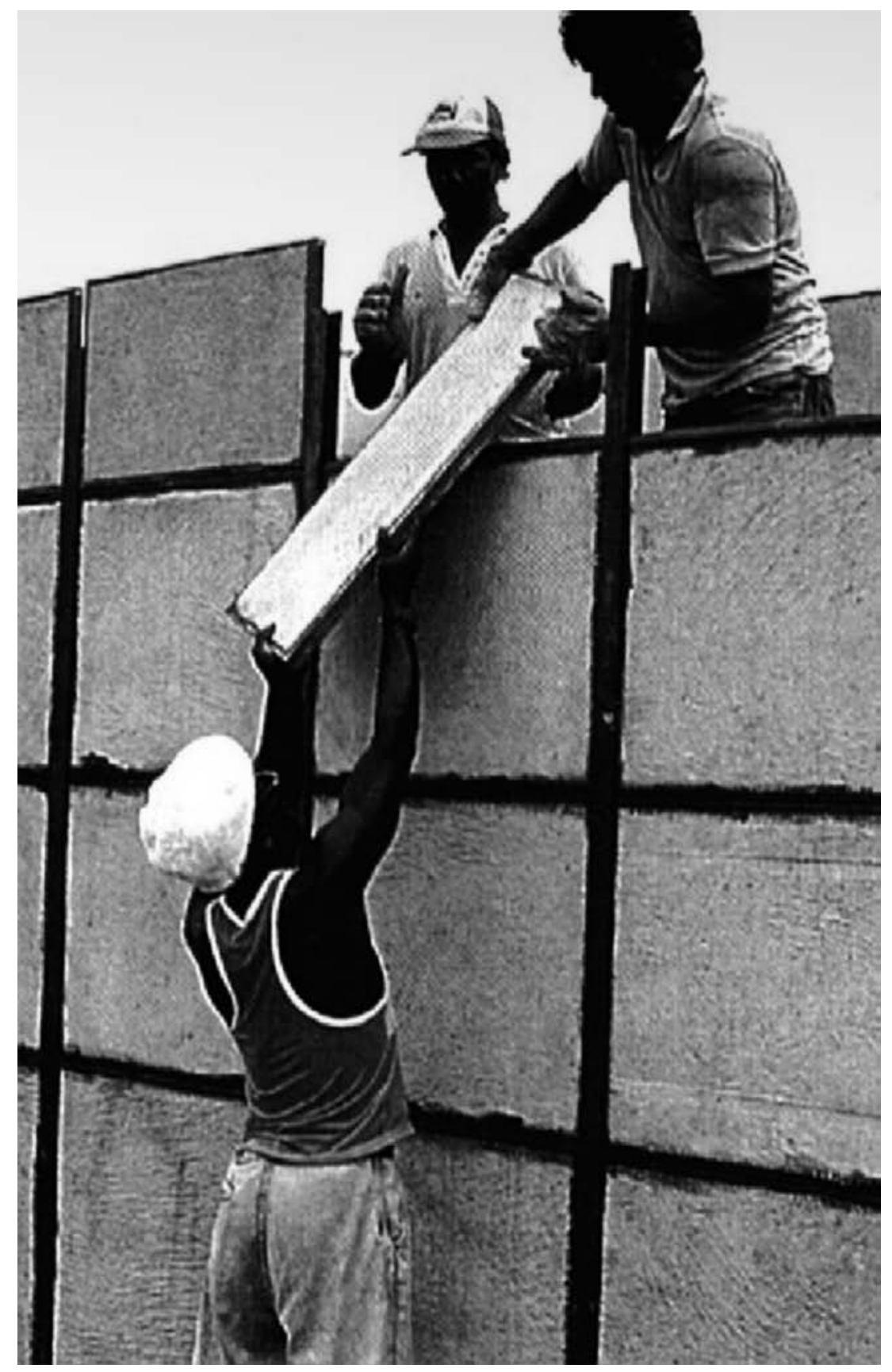

A partir de la crisis del desarrollismo, desde fines de los '70, y afianzado en las décadas siguientes, se instrumenta en América Latina el modelo de globalización de los mercados. Se aplican políticas de ajuste estructural, privatización de servicios y empresas públicas, descentralización de los servicios sociales y las agencias internacionales de crédito acrecientan su gravitación en las decisiones de los gobiernos y los estados nacionales.

El modelo demostró que genera exclusión social, mayores desigualdades entre ricos y pobres y desempleo estructural con su consecuencia en el aumento de la pobreza.

En este contexto, desarrollo es producir más bienes y servicios, cuidando que más gente disfrute de los mis$\operatorname{mos}^{1}$, y pobreza, la carencia de dichos bienes para subsistir e insertarse en el sistema productivo.

Los organismos internacionales de crédito que en un principio orientaban sus acciones a calmar los efectos del ajuste a través de la focalización de los recursos en los sectores de pobreza, capacitación de los recursos humanos involucrados y desarrollo de sistemas de información y previsión integrados, suman en sus recomendaciones al final de los '90 un nuevo concepto: Capital Social, como recurso que podrían utilizar los pobres, carentes del capital económico, para desarrollarse, apuntando a generar las condiciones adecuadas para la creación, conservación y crecimiento de esta forma de capital.

\section{1}

Las primeras referencias al capital social datan de los '60 con Jane Jacobs en su The Death and life of Great American Cities (1961), y en la década del 70 Loury utiliza la expresión para analizar el problema del desarrollo económico de las áreas centrales de las grandes urbes ${ }^{2}$. Pero es a partir de los '80, con Coleman, Bordieu, Putnam y en los debates de los últimos años cuando el concepto comienza a cobrar fuerza en las políticas de los Estados en relación a la pobreza y al desarrollo.

Bordieu (1985) considera que el capital económico constituye la raíz de los demás tipos de capital, incorporando el concepto de capital simbólico asociado al de capital cultural (educación / ilustración, redes sociales, 
1 «La noción de desarrollo integral (de un país, de una comunidad) o segmentadamente (industrial, ambiental, productivo, económico, educativo, social, etc.), es asociada hoy inexorablemente al crecimiento en la capacidad de producir, comprar o poseer (de alguna manera), los bienes y servicios que el mercado nos ofrece».

Bombarolo, F. (1998): Capital social, buscando un horizonte para las politicas y programas de desarrollo en América Latina. Impreso de la maestría en Hyv.

2 Ya Alexis de Toqueville, aún sin nombrarlo, caracterizaba al mismo en su Democracy in America, (s XIX): "Independientemente de las asociaciones permanentes creadas por ley con el nombre de comunas, ciudades y condados, existe una multitud de otras que deben su surgimiento y desarrollo exclusivamente a voluntades individuales [...] Después de la libertad de actuar por sí solo, lo más natural al hombre es la de conjugar sus esfuerzos con los esfuerzos de sus semejantes y obrar en común. El derecho de asociación me parece pues, inalienable por su naturalez así como la libertad individual».

3 Las siguientes definiciones son citadas por Stein, R. (2003) en: Capital Social, desarrollo y políticas públicas en la realidad latinoamericana. Dpto. de trabajo social, Universidad de Brasilia. «...capacidad de los individuos para disponer de recursos escasos en virtud de su pertenencia a redes o estructuras sociales más amplias...» (Portes, 1995); «...simpatía de una persona o grupo hacia otros que puede producir un beneficio, ventaja y tratamiento preferencial para otra persona o grupo de personas más allá de lo esperado en una relación de intercambio...» (Robinson y Siles, 2000); «...normas, redes y relaciones sociales inmersas en las institucione formales e informales de la sociedad, que dan a las personas la posibilidad de coordinar su acción y alcanzar sus metas...» (Narayan, 2001); «...contenido de ciertas relaciones sociales caracterizadas por actitudes de confianza y comportamientos de reciprocidad y cooperación...» (Durston, 2001); prestigio e influencia, poder hacer de los grupos sociales, legitimidad política, etc.), y al capital social al que identifica como relaciones, las cuales se hacen presentes en los hechos sociales fundamentales.

Su preocupación radica en evaluar la manera en que estos últimos se subordinan al capital económico y cómo interactúan con estructuras más amplias que producen las desigualdades sociales.

Coleman (1990) define al capital social como un recurso que se acumula y se amplía en la medida en que se utiliza, producido por la interacción de una variedad de entidades diferentes con características comunes: todas se fundan en algún aspecto de la estructura social y las acciones de los individuos que pertenecen a esa estructura, son facilitadas por la posesión del mismo.

Así, el capital social se presenta tanto en el plano individual como en el colectivo, contenido en las personas y en su estructura de relaciones, constituyéndose en un recurso social informal que las une y les impone reglas y sanciones.

Putnam (1993) destaca como elementos constitutivos del capital social la confianza, la reciprocidad y la cooperación, reconociendo que el contexto social y la historia condicionan el desempeño de las instituciones.

Que el sistema democrático promueve valores como igualdad, equidad y solidaridad, no puestos en práctica totalmente en la mayoría de las democracias occidentales, por lo que sugiere la máxima descentralización posible del poder del Estado, el rescate la cultura organizacional local y el fortalecimiento de las organizaciones de la sociedad civil.

Valora la creación y refuerzo de redes de confianza y trabajo conjunto entre estas instituciones y menciona dos ejemplos en los que el capital social aporta a la construcción de un desarrollo más sustentable y democrático: la creación de mecanismos formales de control social y la generación de sistemas de créditos rotativos para personas de bajos ingresos (economía solidaria). En los últimos años de la década pasada, hubo numerosos aportes ${ }^{3}$ ante la importancia asignada al concepto por parte de organismos internacionales vinculados al desarrollo.
Para la CEPAL ${ }^{4}$ el Capital Social es «el conjunto de normas, instituciones y organizaciones que promueven la confianza y la cooperación entre las personas, las comunidades y la sociedad en su conjunto", asociándolo a lo conductual y diferenciándolo del capital cultural, al que hace corresponder al ámbito abstracto y normativo.

En los documentos de este organismo, se establece una diferencia entre el capital social individual y el comunitario, definiendo al primero como la confianza y la reciprocidad que se extienden a través de redes a las que denomina ego-centradas. El segundo es aquel que se expresa en instituciones complejas, con contenido y gestión.

Considerando el nivel territorial, se identifican distintos tipos de redes de capital social, ya sea individual o comunitario, que suponen distintas formas de funcionamiento particular en las relaciones sociales: individual, grupal, sistemas institucionales comunitarios (juntas de vecinos, con liderazgos y control social de sus miembros), conexiones distantes horizontales y verticales (organizaciones asociativas de segundo nivel).

«En esta acepción, el capital social reside, no en las relaciones interpersonales sino en sus estructuras normativas, gestionarias y sancionarias. En ambos casos, sin embargo, la noción de red (como sustrato de la asociatividad) juega un rol significativo", y coincidiendo con Putnam, se hace referencia a la posibilidad de crearse un «círculo virtuoso» capaz de generar equilibrio social, lo que implica la necesidad de existencia de relaciones horizontales y espontáneas de cooperación, confianza, reciprocidad, como así también de relaciones formales con el Estado y el sector privado.

También se destaca su importancia como recurso, y por lo tanto, acumulable, transferible y aplicable a fines productivos, lo que fundamenta la metáfora de la asociatividad tratada como «capital».

\section{2}

El Banco Mundial, ${ }^{5}$ en sus recomendaciones, destaca la importancia de las relaciones con el Estado y el sector privado. Reconociendo el valor de «una serie de asociaciones horizontales entre personas que incluyen redes 

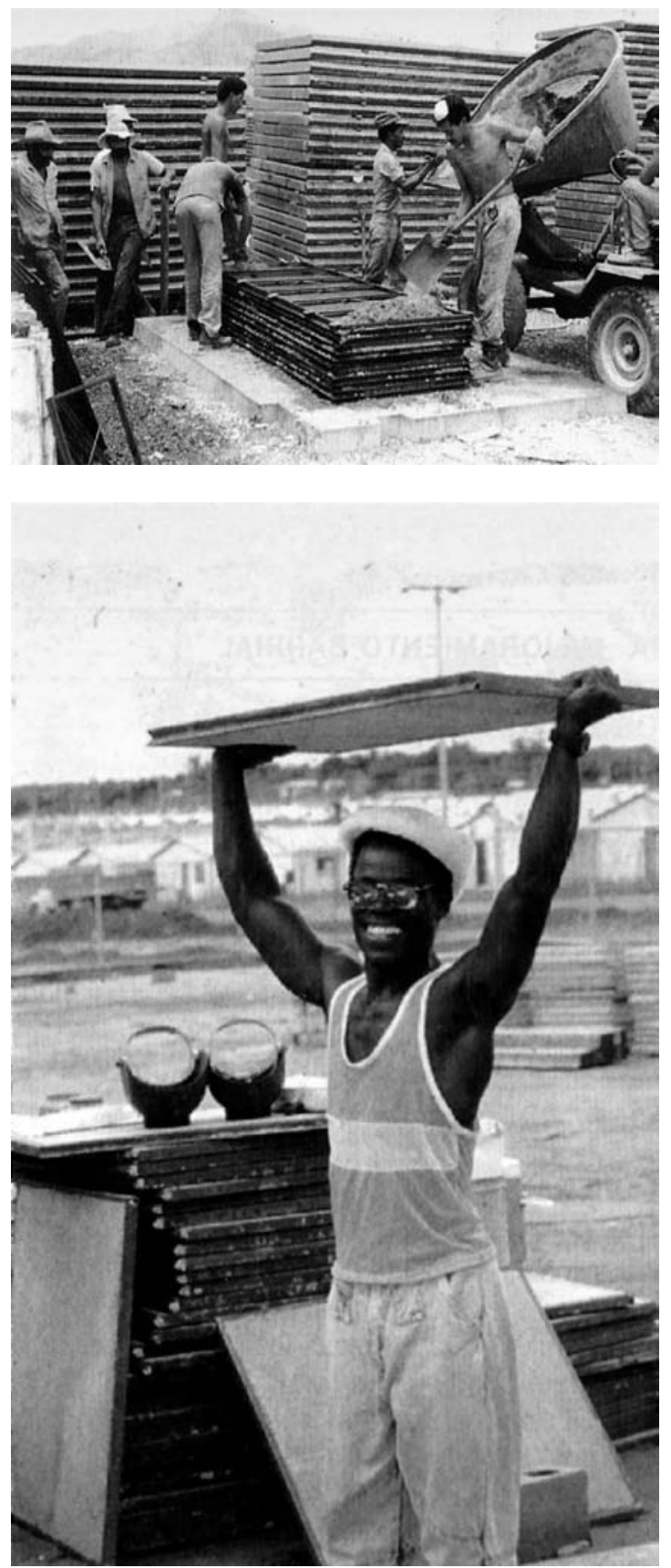

sociales y normas asociadas que afectan a la productividad y el bienestar de la comunidad», hace la salvedad que esto puede operar desventajosamente cuando las comunidades o grupos están aislados o tienen intereses exclusivamente locales, citando como ejemplos redes de corrupción o cárteles de la droga.

De este modo «las relaciones horizontales son necesarias para dar un sentido de identidad y un propósito común a las comunidades, pero... sin formar relaciones que trasciendan varias divisiones sociales (por ejemplo religión, etnia, estatus socio-económico), las relaciones horizontales pueden convertirse en una base para la búsqueda de intereses restringidos, que impidan el acceso a la información y a los recursos materiales que de otra manera pudieran ser de gran asistencia para la comunidad (por ejemplo, información sobre vacantes de trabajo o acceso al crédito).»

Reforzando más aún estos conceptos: «...la capacidad de varios grupos sociales para actuar en su propio interés depende crucialmente del apoyo (o la falta de apoyo) que reciban del Estado y del sector privado. De la misma manera, el Estado depende de la estabilidad social y del apoyo popular. En resumen, el desarrollo económico y social prospera cuando los representantes del Estado, del sector empresarial y de la sociedad civil crean foros a través de los cuales puedan identificar y alcanzar metas comunes».

El BM va más allá en sus recomendaciones, sugiriendo los modos de operacionalizarlas sustentándose en estudios realizados en distintas comunidades con el objeto de encontrar un modo de medición del capital social. Aclara que «no es posible ni deseable lograr una única y verdadera medida» y que una posibilidad es combinar tipos y metodologías de investigación cuantitativas, comparativas y cualitativas:

Tomando como base las recetas convencionales para mejorar las perspectivas económicas de las comunidades y naciones que incluyen mejorar la educación y la salud, construir instituciones políticas competentes y responsables y facilitar la aparición de mercados libres que puedan competir en la economía global, entiende que el 
«...normas o valores compartidos que promueven la cooperación social [...], permite que los individuos débiles se agrupen para defender sus intereses y organizarse en apoyo de sus necesidades colectivas...» (Fukuyama, 2001). 4 Cepal (2001): «Capital social y pobreza». Conferencia regional sobre capital social y pobreza Santiago de Chile, 09/2001.

5 Banco Mundial, documentos varios.

6 Bombarolo, F. (1998), op. cit. mejoramiento de la educación y de la salud está directamente vinculado con el nivel de involucramiento en el seguimiento y control de sus actividades por parte de padres y ciudadanos, que para que las instituciones sean competentes y responsables se deben tener en cuenta las formas preexistentes de capital social de las comunidades (afirmación que debería contextualizarse en el marco de un ejemplo citado, el de Rusia cuando instrumentó la privatización de empresas estatales provocando, a juicio del BM, desigualdades y violación de los derechos humanos). En relación a los mercados, afirma que como una de las características definitorias de ser pobre es la falta de conexiones con la economía formal, se debe "ayudar a los pobres a trascender sus redes de seguridad» familiares y vecinales a fin de que puedan tener acceso a recursos adicionales, ya que el capital social de los ricos es el que le permitirá promover sus intereses. También destaca el valor de la participación de los pobres en el diseño y ejecución de los proyectos de desarrollo. Esto «...ayuda no sólo a producir proyectos más apropiados sino también asegura que dichos proyectos estén mejor enfocados para beneficiar a aquellos que tienen mayores necesidades» (Narayan, 1995), sustentándose en estudios que concluyen en que la inclusión del pobre desde el comienzo ayuda a crear más confianza y lealtad hacia el proyecto.

Fija la atención en la necesidad de conectar el capital social con iniciativas intersectoriales. Al referirse al sistema de microcréditos colectivos del Banco Grameen de Bangla Desh, lo evalúa como una primera instancia a ser complementada con redes sociales más amplias donde puedan converger los intereses del sector público, el privado y de la sociedad civil en su conjunto.

Por último, hace referencia a la importancia de facilitar el acceso de los pobres a la infraestructura de las comunicaciones que permitan crear «foros en los cuales distintos grupos de intereses pueden establecer relaciones unos con otros» y que puedan contribuir al bienestar comunitario.

Resumiendo, «El capital social supone, entonces, una revalorización del concepto de los '80 de fortalecimien- to institucional de las organizaciones sociales [...], que implica apoyo a la formación de redes, espacios y mecanismos que permitan mejorar las decisiones entre las instituciones y actores que tienen un interés común (stake-holders)» ${ }^{6}$.

3

Lo aquí expuesto permite establecer algunas conclusiones:

1. El concepto de Capital Social es presentado como relevante por el mayor protagonismo que asume la sociedad civil en su relación con el Estado y las políticas públicas.

2. Se ponen de relieve efectos positivos tales como control social, creación de confianza entre individuos, cooperación coordinada, resolución de conflictos, movilización y gestión de recursos comunitarios, legitimación de líderes, generación de ámbitos de trabajo, prevención y sanción de quienes abusan de él y producción de bienes públicos.

3. La diferenciación de las formas individuales y comunitarias de capital social, permite identificar con mayor precisión implicaciones de políticas para los gobiernos, para el sector privado y para las organizaciones de la sociedad civil, definiéndose los beneficios de la interacción de los sectores diversos de la sociedad con distintos grados de poder.

En la instrumentación de estos lineamientos, es decir, en el planeamiento y puesta en marcha de los mismos a través de programas concretos hay algunas cuestiones que se podrían observar:

Un primer aspecto es el de la participación. Existen metodologías para la planificación y gestión participativas que se utilizan en los programas, a través de las cuales se convoca al trabajo mancomunado de funcionarios, técnicos, organizaciones y potenciales beneficiarios. En el mejor de los casos existen instancias de análisis y debates con una transferencia limitada de cuotas de poder para la toma de decisiones. Pero, por el hecho de ser solo metodologías pueden servir tanto para generar nuevos canales de creación colectiva como para cristalizar las visiones tradicionales dominantes sobre desarrollo y pobreza. 
Caldarelli y Rosenfeld entienden la participación como «...un proceso complejo desencadenado por quienes comparten y priorizan necesidades constituidas como demandas, que se encamina a influir en diseños institucionales, en definición de políticas y programas, en definitiva, en cambios en la estructura del poder». Así, la problemática de la participación sería la «...construcción de áreas de igualdad, en un contexto de intereses desiguales y heterogéneos»?

Refiriéndose a la gestión participativa, Pelli la define como «una asociación asimétrica con el cometido de lograr (o, de modo más realista, de tender a) una situación de simetría» ${ }^{8}$.

La asimetría está fundada en los modos en que está organizada la distribución del poder entre los actores de la sociedad. Tender a una situación de simetría implica lograr una redistribución del mismo de un modo más equilibrado, transferir cuotas de poder desde los sectores que lo detentan hacia otros que carecen del mismo, observándose dos carencias básicas que sufren estos sectores: la del poder de gestión y negociación, y la de inserción social satisfactoria o equitativa.

«Estas carencias, que se muestran tan degradantes como la de recursos de supervivencia inmediata (bienes y servicios), aunque las relaciones de causa-efecto sean menos evidentes puede leerse también como excesiva desvalorización dentro del juego de transacciones de la sociedad formal, tanto en el ámbito social global y estructural como en el de los contactos personales intersectoriales de todos los días: trabajo, prestación y recepción de servicios, simple convivencia lado a lado..." Un segundo aspecto que se destaca en las recomendaciones de los organismos multinacionales es la importancia asignada al fortalecimiento de las organizaciones de la sociedad civil. En este campo, se apunta a dos áreas de apoyo: a las ONGs y a las Organizaciones Territoriales de Base, entendiendo a estas últimas como «...conjuntos de individuos que comparten una misma localización
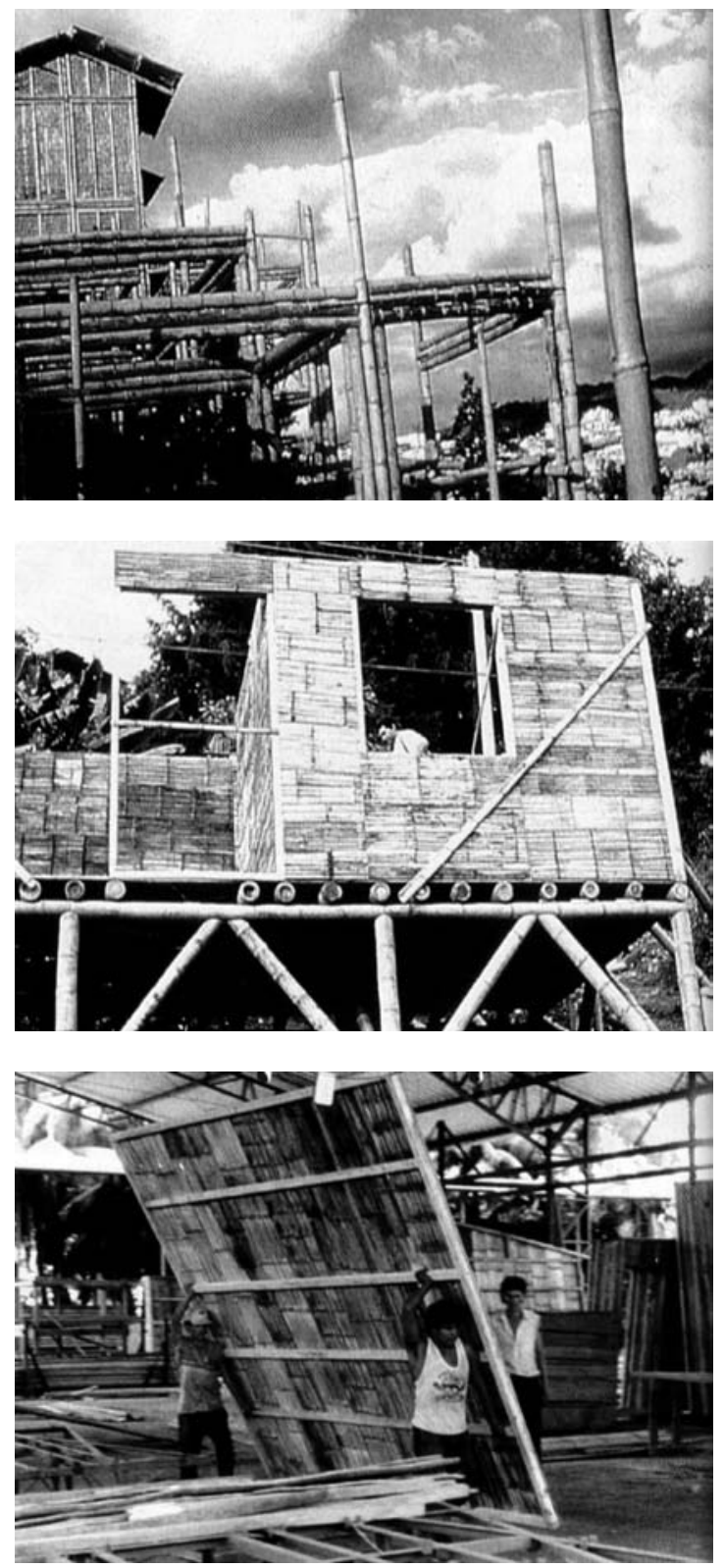
territorial (comúnmente), con relaciones sociales formalizadas, determinadas culturalmente, vinculadas a la resolución colectiva de necesidades materiales y simbólicas de la vida cotidiana y que disponen de un modo de funcionamiento interno y de roles diferenciados...» ${ }^{10}$ Houtart ${ }^{11}$ identifica a la sociedad civil como el lugar donde se desarrollan las potencialidades del individuo, el espacio de las libertades. La libertad de empresa es una de ellas y a su vez fuente de todas las otras.

Así, la empresa es el pilar fundamental y a ésta se articulan las instituciones que juegan su papel en la reproducción social: escuela, iglesia, etc. Con esta concepción «...reforzar la sociedad civil significa favorecer la libertad de empresa, dinamizar los actores sociales empresariales, reducir el lugar del Estado y finalmente reproducir la relación social que asegura la dominación de clase, hoy mundializada».

Estratégicamente, puede constituirse como una red de instituciones u organizaciones voluntarias no contestatarias, religiosas o laicas, orientadas principalmente a acciones de alivio de la pobreza. El papel del Estado sería proporcionar marco jurídico a la propiedad privada y funcionamiento de la libre empresa, asegurar el funcionamiento de la reproducción social y proteger a los individuos.

«La Sociedad Civil se despolitiza, pues frente al mercado la política es cada vez más virtual. Los movimientos sociales buscan su identidad exclusivamente en su propio campo, en ruptura con la tradición política. Ciertas ONGs cultivan una ideología ferozmente anti-estado. Los movimientos religiosos se multiplican centrados en la salvación individual y desprovistos de proyección social».

Un enfoque alternativo sobre la sociedad civil podría expresarse como «...el espacio donde se construyen las desigualdades sociales y existen en su seno instituciones y organizaciones que representan intereses de clase muy divergentes». Comprende todo tipo de organización constituida desde el reconocimiento de la estructura de clases que opera en los intersticios del sistema, plan- 7 Caldarelli; Rosenfeld (2001),

teando modos alternativos de acción sobre las necesida- cit. p/ Bombarolo, F. (1998), op. cit. des de la sociedad.

«No es capacitar a las organizaciones para que asuman la responsabilidad de hacer frente a sus problemas, sino generar organizaciones fuertes para poder negociar, hacer valer sus derechos y equilibrar la balanza del poder (económico y simbólico) exageradamente inclinada hacia un sector muy pequeño de la sociedad» ${ }^{12}$.

Un tercer aspecto es el de tejido social o redes de trabajo, donde el BM parece concentrar los mayores esfuerzos: «nunca es exagerado resaltar la importancia de la red de confianza y la asociación para el desarrollo sostenible» ${ }^{13}$. En la práctica, el Banco plantea una política de alianza comprendida como iniciativas conjuntas del sector público con el privado.

El concepto está influido por el de alianza estratégica, que proviene del mundo de los negocios y que ha dado sustento a las operaciones intersectoriales (planeamiento estratégico).

La idea detrás de estas estrategias es la de sinergia: «... significa el comportamiento de un sistema completo, que resulta impredecible a partir del comportamiento de cualquiera de sus partes tomada aisladamente [...], la sinergia connota una forma de potenciación, es decir, un proceso en el que la potencia de los elementos asociados es mayor que la potencia sumada de los elementos tomados aisladamente» ${ }^{14}$.

Al respecto, Stein observa que en lugar del círculo virtuoso propuesto por la CEPAL a partir de las investigaciones de Putnam, «éste todavía parece distante de verse configurado, la fuerza y el movimiento del círculo vicioso solo permiten identificar experiencias aisladas, caracterizándose más por la reiteración del discurso, aparentemente nuevo, que por la capacidad de hacerlo efectivo en la práctica». ${ }^{15}$

Entonces «el desarrollo viene a ganar adjetivos (humano, humano sostenible, integrado, etc.) que buscan suavizar como objetivo de las políticas habitacionales.

9 Pelli, V. (1997), op. cit. 10 Alderete (1995), cit. p/ Bombarolo, F. (1998), op. cit. Houtart, F. (s/D): Hacia una Sociedad Civil globalizada: la de abajo o la de arriba. Centro Tricontinental, Lovaina la Nueva. Foro Mundial de las Alternativas.

12 Bombarolo, F. (1998), op. cit. Banco Mundial (2000),

cit. p/ Stein, R. (2003), op. cit. 14 Max-Neef,

cit. p/ Bombarolo, F. (1998), op. cit. Stein, R. (2003), op. cit. 
16 Stein, R. (2003), op. cit. 17 De la Maza, ironiza sobre este aspecto: «es posible establecer 1 hipótesis que en el marco de las llamadas "reformas de segunda generación”, en los países de América Latina, que apuntan al rediseño institucional, manteniendo un Estado reducido, la posibilidad de fortalecer la capacidad de los ciudadanos por encarar los problemas que el sector público ha abandonado y hacerlo a bajo costo resulta una perspectiva atractiva». los efectos de su definición en lo tocante a la distribución desigual de los bienes producidos colectivamente y su provecho por una minoría» ${ }^{16}$.

Esta visión de desarrollo es la que anima las recomendaciones y acciones de los sectores neoliberales representados por los organismos internacionales de crédito, acompañados con Estados disminuidos y despojados de responsabilidades y recursos, cuyo rol parece ser el de modelar desde la superestructura una sociedad despolitizada y orgánica, y socializada por vecindad geográfica ${ }^{17}$.

\section{Conclusión}

Los lazos de confianza, solidaridad y cooperación, existen y han existido siempre en las sociedades: a nivel familiar, vecinal e incluso regional ante situaciones de crisis.

La cuestión es, si estas relaciones pueden constituirse en un recurso para el desarrollo. o, más aún, si el desarrollo en los sectores desposeídos puede sustentarse en la institucionalización en horizontal y en vertical de estas relaciones, sin operar en algún otro tipo de medidas correctivas, por ejemplo, sobre las leyes del mercado.

Hay autores que defienden una visión alternativa a la de los organismos: el poder hacer de los grupos está fundado en la existencia de un capital simbólico que puede ser obtenido a lo largo de un proceso de institucionalización y la eficacia simbólica depende del grado en el que la visión propuesta está fundada en la realidad, es decir, en las afinidades objetivas entre las personas que se trata de juntar. En esta perspectiva adquiere relevan- cia la dimensión política, al reconocerse la existencia del conflicto social y es a través de la participación en los espacios que brinda el sistema democrático es donde se deben dirimir las diferencias y dicho conflicto.

Bajo éste enfoque, el capital social no es ajeno a los conflictos sociales existentes, implica un tipo de poder, que junto con el económico, determina la ubicación de las personas y grupos dentro del espacio social, significa capacidad para negociar, decidir, imponer criterios y estilos de convivencia y necesariamente está vinculado al poder político.

Houtart expone algunas ideas que se resumen a continuación:

Debería apuntarse a lograr una acción sistemática que reagrupe a todos aquellos que desde distintos dominios de la vida colectiva contribuyen a construir una economía, una política y una cultura diferentes, y que puedan elaborar su propia agenda con independencia de los medios de decisión mundiales.

Que sean portadores de utopías, en el buen sentido del término, que puedan inspirar y motivar los compromisos sociales. Que se esté a la búsqueda de alternativas a todos los niveles, grandes conquistas políticas y también en la vida cotidiana.

Y fundamentalmente, que se apunte a la conquista de los espacios públicos, esto es, articulación con la política. El desafío, entonces, sería la construcción de una relación de fuerzas que permita apropiarse de cuotas de poder para una toma más autónoma de decisiones. 


\section{Bibliografía}

Atria, R. (2002): Capital social y pobreza: asociatividad y capacidad de movilización en las estrategias de reducción de la pobreza.

Banco mundial, (s/D): «;Qué es exactamente la desigualdad?» Sitio web de Poverty net.

Banco mundial, (s/D): «¿Qué es el capital social?» Sitio web de Poverty net.

Bombarolo, F. (s/D): Sin salida (aparente...). Fundación Poleas.

Bombarolo, F. (1998): Capital social, buscando un horizonte para las políticas y programas de desarrollo en América Latina. Impreso de la maestría en Hyv.

CePal, Capital social y pobreza, (2001). Documento preparado para la conferencia regional sobre Capital social y pobreza, realizada en Santiago de Chile los días 24 a 26 de setiembre de 2001.

De la Maza, G. (200o): Sociedad civil y construcción de capital social en América Latina: ¿hacia donde va la investigación?

Houtart, F. (s/D): Hacia una sociedad civil globalizada la de abajo o la de arriba. Centro Tricontinental, Lovaina la Nueva, Foro Mundial de las Alternativas.

Pelli, V. (1997): La integración social como objetivo de las políticas habitacionales. Impreso de la maestría en Hyv.

Stein, R. (2003): Capital social, desarrollo y políticas públicas en la realidad latinoamericana.

Dpto. de Trabajo Social, Universidad de Brasilia. 